\title{
Abrupt turnover in calcareous-nannoplankton assemblages across the Paleocene/Eocene Thermal Maximum: implications for surface-water oligotrophy over the Kerguelen Plateau, Southern Indian Ocean
}

\author{
Shijun Jiang and Sherwood W. Wise, Jr. \\ Department of Geological Sciences, 108 Carraway Building, Florida State University, Tallahassee, FL 32306-4100, USA (jiang@gly.fsu.edu; \\ wise@gly.fsu.edu)
}

\begin{abstract}
Ocean Drilling Program (ODP) Core Section 183-1135A-25R-4 from the Kerguelen Plateau in the Indian Ocean sector of the Southern Ocean represents only the second complete, expanded sequence through the Paleocene/Eocene Thermal Maximum (PETM; 55 Ma) recovered from Antarctic waters. Calcareous nannoplankton at this site underwent an abrupt, fundamental turnover across the PETM as defined by a carbon isotope excursion. Although Chiasmolithus, Discoaster, and Fasciculithus exponentially increase in abundance at the onset, the former abruptly drops but then rapidly recovers, whereas the latter two taxa show opposite trends due to surface-water oligotrophy. These observations confirm previous results from ODP Site 690 on Maud Rise. The elevated $p \mathrm{CO}_{2}$ that accompanied the PETM caused a shoaling of the lysocline and carbonate compensation depth, leading to intensive dissolution of susceptible holococcoliths and poor preservation of the assemblages. Similarities and contrasts between the results of this study and previous work from open-ocean sites and shelf margins further demonstrate that the response to the PETM was consistent in open-ocean environments, but could be localized on continental shelves where nutrient regimes depend on the local geologic setting and oceanographic conditions.
\end{abstract}

Citation: Jiang, S., and S.W. Wise (2007), Abrupt turnover in calcareous-nannoplankton assemblages across the Paleocene/Eocene Thermal Maximum: implications for surface-water oligotrophy over the Kerguelen Plateau, Southern Indian Ocean, in Antarctica: A Keystone in a Changing World - Online Proceedings of the $10^{\text {th }}$ ISAES, edited by A. K. Cooper and C. R. Raymond et al., USGS Open-File Report 2007-1047, Short Research Paper 024, 5 p.; doi:10.3133/of2007-1047.srp024.

\section{Introduction}

One of the major paleoclimate discoveries of the scientific Ocean Drilling Program (ODP) was the Paleocene/Eocene Thermal Maximum (PETM) at Maud Rise (ODP Site 690) in the Southern Ocean (SO; Kennett and Stott, 1991; Bralower, 2002). We report here a second such site located half way around the Antarctic continent in the SO at ODP Site 1135 on the Kerguelen Plateau (KP; Fig. 1).

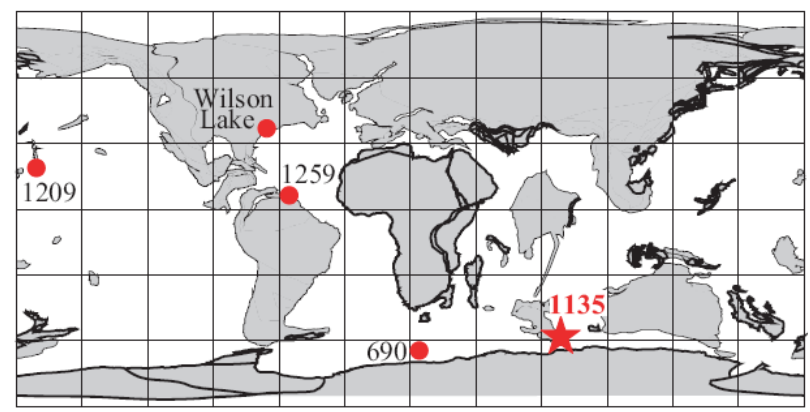

Figure 1. ODP Site $1135(\star)$ and other reference sites $(\bullet)$ on an ODSN 55-Ma plate reconstruction.

The PETM was a catastrophic, rapid greenhouseforced global warming event $\sim 55$ m.y. ago (surface- and bottom-water temperatures rose by $\sim 5^{\circ} \mathrm{C}$ at high latitudes within <22 k.y.; [Kennett and Stott, 1991; Norris and Röhl, 1999; Zachos et al., 2003]). The environmental perturbations associated with the PETM triggered an abrupt overturn in ocean chemistry (Dickens et al., 1995; Zachos et al., 2005) and circulation (Kennett and Stott, 1991; Nunes and Norris, 2006). The concomitant biotic turnovers include a mass extinction $(30 \%-50 \%)$ of benthic foraminifers, an evolutionary radiation of land mammals and vegetation, a diversification of the planktonic foraminifers, as well as the evolution of ephemeral "excursion taxa" among planktonic foraminifera and nannofossils (see Kelly et al. [1996], Aubry et al. [1998] and references therein).

A global, negative carbon-isotope excursion (CIE) of $\sim 3 \%$ that accompanied the PETM is most parsimoniously explained by a massive release of sedimentary methane hydrates to the ocean-atmosphere systems (Dickens et al., 1995). Alternatively, the carbon that fueled the PETM may have been derived the thermal cracking of methane in coals in the North Atlantic (e.g., Svensen et al., 2004; Storey et al., 2007). Either way, methane, or its subsequent oxidation product, carbon dioxide, would have immediately contributed to greenhouse warming and changes in ocean chemistry.

Despite its global extent, sedimentary archives of the PETM are limited and imperfect. Low-latitude PETM records generally contain a dissolution interval barren of calcareous fossils; hence we cannot say from those nannofossil records what happened during the first few k.y. of the event. Our high-latitude records, however, have yielded calcareous fossils, although at ODP Site 1135 they are altered by diagenesis (e.g., fragmentation, dissolution, and overgrowth) that to some degree hinders the correct identification of species and even genera.

Surface-dwelling plankton appear to have responded to the PETM environmental changes in fundamentally different ways from benthic organisms, but their strategies remain poorly understood due to the limited number of high-resolution investigations using 
calcareous nannofossils (e.g., Monechi et al., 2000; Bralower, 2002; Gibbs et al., 2006) and geochemical proxies (e.g., $\mathrm{Sr} / \mathrm{Ca}$ ratios and biogenic Barium accumulation rates [Bains et al., 2000; Stoll and Bains, 2003]).

Contrasts among previous studies in the equatorial Atlantic, SO, New Jersey continental margin, and central paleoequatorial Pacific (Fig. 1) suggest that local differences in geologic setting and oceanographic conditions influence the biotic response to the PETM (Bralower, 2002; Gibbs et al., 2006; Jiang and Wise, 2006). Controversy over the phytoplankton's response to the PETM even arises from different productivity proxies at the same locality on Maud Rise in the SO (Bains et al., 2000; Bralower, 2002; Stoll and Bains, 2003). In order to determine the actual impact of the PETM on surfacewater dwellers at high southern latitudes, we evaluated high-resolution variations in nannofossils and bulk stable isotopes at Site 1135 in $1567 \mathrm{~m}$ of water on the KP (Fig. 1 ), a sequence that was first studied in a low-resolution study by Arney (2002).

\section{Materials and methods}

ODP Core Section 183-1135A-25R-4 is a carbonaterich nannofossil and foraminifer ooze, selected for this study because of the coherence and completeness of the portion recovered. Another PETM section was recovered farther south on the KP at Site 738, but recovery was not as complete and no detailed assemblage work has been done on that section (Bralower, 2007, pers. comm.). Samples were taken at a 2-cm spacing throughout the core section. Slide preparation followed the modified "glass-bead" technique of Okada (1992), whereas taxonomic concepts follow Bown (2005); techniques to determine percentage abundance were adapted from Jiang and Wise (2006). Assemblage preservation was quantified using the ratio of identifiable specimens to indeterminate isolated nannofossil shields. Five to ten bulk foraminifers from the $63-\mu \mathrm{m}$ fraction were analyzed on a Finnigan Mat Delta plus XP mass-spectrometer in the National High Magnetic Field Laboratory (NHMFL) at FSU.

\section{Results: variations in nannofossil assemblages}

ODP Core Section 183-1135A-25R-4 represents a stratigraphically complete, expanded sequence across the PETM as defined by the extent of the CIE (Fig. 2), and contains poorly preserved nannofossil assemblages. The severe diagenetic alteration renders most specimens identifiable only at the generic level, although important ecological indicators such as Chiasmolithus, Discoaster, Fasciculithus, Sphenolithus, and Zygrhablithus, survived due to their robust construction. Biscutum and Hornibrookina are also readily distinguishable when present.

Unidentifiable isolated nannofossil shields, and to a lesser extent, Toweius, Coccolithus pelagicus, and Zygrhablithus bijugatus dominate assemblages (Fig. 2).
The isolated shields are quite abundant at the onset of the PETM, drastically decrease after the CIE peak, increase somewhat during the CIE's recovery, then decrease upsection. Zygrhablithus bijugatus shows a steady decrease in abundance from the onset to the peak of the CIE, then abruptly increases thereafter, but remains high and increases again at the top of the section.

The ecological indicators demonstrate significant stratigraphic variations. Discoaster, Fasciculithus, and Sphenolithus exponentially increase in abundance from the onset of the PETM, and peak prior to the CIE peak, at which point they decrease abruptly and then remain consistently low thereafter. The abundance of Chiasmolithus also demonstrates an exponential increase at the onset, abruptly drops to its lowest value prior to the CIE peak, then mimics the exponential recovery of the CIE, but decreases again before the CIE's full recovery. Biscutum and Hornibrookina occur sporadically in the section, present with abundant Chiasmolithus but at low levels or absent when Chiasmolithus abundance is low.

\section{Discussion}

\section{Stable isotope pattern}

The onset of the CIE occurs within an 18-cm interval before the peak is reached (Fig. 2). In most deep-sea sections, this onset usually occurs in 1 or $2 \mathrm{~cm}$ of core. This, we believe, is the result of high sedimentation rates at this relatively shallow oceanic site, as evidenced by the high numbers of dissolution-susceptible holococcoliths (Z. bijugatus) preserved throughout the sequence. Thus, this sequence appears to be ideal for high-resolution studies. The full course of the CIE exhibits a more or less symmetrical "V" shape in our data, indicating that the release of light carbon was a gradual, single injection, instead of multiple pulses as suggested by Bains et al. (1999).

\section{Ecological implications of assemblage variations}

Covariations among nannofossil abundances and their corresponding correlation coefficients highlight taxa with similar trends, suggesting likely ecological groupings and possibly similar preservation states (Fig. 2; Table 1). Based on their biogeography and relationships with paleoenvironmental proxies, Discoaster, Fasciculithus, and Sphenolithus are considered as K-selected specialists adapted to warm, oligotrophic waters (e.g., Haq and Lohmann, 1976; Wei and Wise, 1990; Chepstow-Lusty et al., 1992); whereas r-mode taxa include Chiasmolithus, Biscutum, and Hornibrookina thriving in cool, eutrophic conditions (e.g., Wei and Wise, 1990; Wei and Pospichal, 1991; Bralower, 2002). Nannofossil assemblages show significant variations within the section. These variations cannot be an artifact of selective dissolution, based on the fact that 1) there is a high negative correlation between $\mathrm{K}$ - and r-selected taxa in the lower section with 


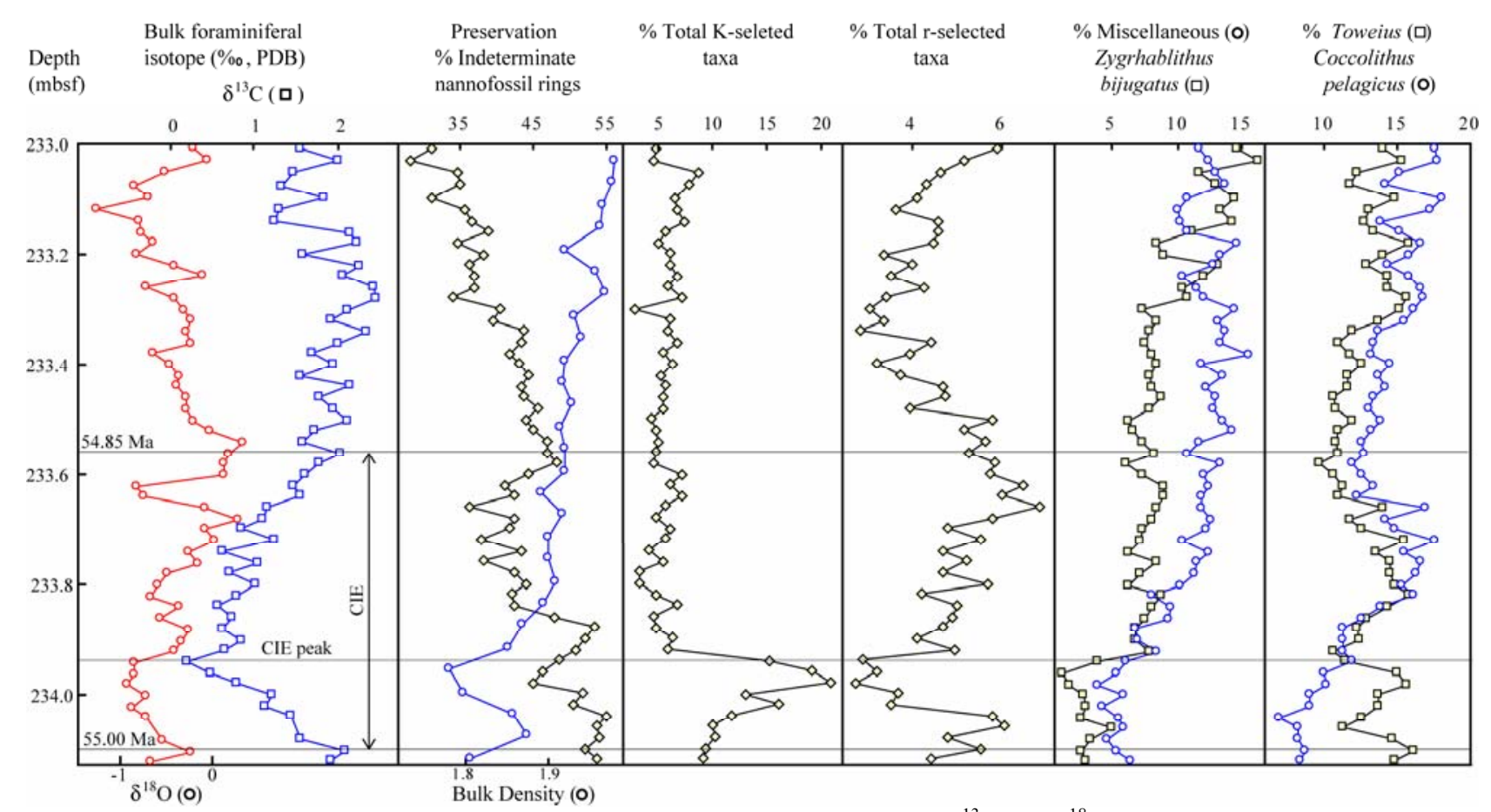

Figure 2. Percentage abundances of nannofossil groupings and bulk foraminiferal $\delta^{13} \mathrm{C}$ and $\delta^{18} \mathrm{O}$ across the PETM from ODP Site 1135. K-selected taxa include Discoaster, Fasciculithus, and Sphenolithus, while r-selected taxa include Biscutum, Chiasmolithus, and Hornibrookina. Age assignments followed Zachos et al. (2003) based on the apparent duration of the CIE. Bulk density data are from the ODP Janus Database. Variations in percentage of unidentifiable isolated nannofossil shields generally depict the preservation of fossil assemblages. Note different abundance scales. mbsf $=$ meters below sea floor.

the low abundances of Z. bijugatus (a dissolutionsusceptible holococcolith), and 2) r-selected taxa fluctuate while K-selected taxa do not in the upper section where Z. bijugatus consistently increases (Fig. 2). Otherwise there would be a consistent relationship between these two groups. Variations in preservation may in part account for the fluctuations; however, environmental factors are likely responsible for the abrupt changes across the PETM.

At Site 1135, abundances of K-selected taxa covary and show opposite tends to r-mode specialists (Fig. 2). Although both nutrient availability and temperature exerted an influence on the abundance increases in these K-selected taxa prior to the peak of the PETM, their abrupt decrease thereafter coincides with a warming trend of $8^{\circ} \mathrm{C}$ in the high latitudes (Kennett and Stott, 1991). This suggests that nutrient availability exerted a stronger influence at this site.

As Z. bijugatus, a readily distinguishable holococcolith even in poorly preserved materials, is susceptible to dissolution (see Crudeli et al., 2006 and references therein), it is therefore a sensitive indicator of seawater $\mathrm{pH}$ values induced by the rapid release of 1500 2200 gigatons of methane (Dickens et al., 1995). The consequent effects of corrosion diminished nannofossil preservation, raising the number of indeterminate isolated nannofossil shields and fragments during the development of the CIE. These decreased, however, as the system reverted to pre-event conditions.

\section{Nannoplankton productivity changes across the PETM in the Southern Ocean}

The variations in nannofossil assemblages at Site 1135 primarily reflect surface-water productivity presumably with little changes in salinity as inferred from the complementary SO Site 690 (Kennett and Stott, 1991). Despite a progressive warming towards the peak of the PETM inferred by decreasing $\delta^{18} \mathrm{O}$ values, the overall assemblage changes do not reflect a steady trend toward oligotrophy, but rather signify eutrophication at the onset as indicated by the exponential increase of $r$ mode taxa in contrast to the negligible increase of kselected specialists (Fig. 2). Similar precursor events just prior to the onset of the PETM were also observed at ODP Sites 690 and 1259 (Bralower, 2002; Jiang and Wise, 2006, respectively); the cause, however, remains unknown, although this eutrophic condition could be a spontaneous response of the destabilized ocean circulation system upon reaching or crossing its threshold value.

Thereafter, the abrupt decrease in abundance of $r$ selected taxa and concomitant increase of K-selected specialists denotes a fundamental oceanographic turnover that produced an oligotrophic condition. This result is quite consistent with that from Site 690 (Bralower, 2002), suggesting that surface-water oligotrophy prevailed in the southern high latitudes. Contrasts between these results with other studies at Site 690 using geochemical and benthic assemblages have 
Table 1 Calcareous nannofossil distribution in ODP Hole 1135A

\begin{tabular}{|c|c|c|c|c|c|c|c|c|c|c|c|c|c|c|c|c|c|c|c|c|c|c|c|c|c|c|c|c|c|c|c|c|c|c|}
\hline $\begin{array}{c}\text { Core, Section, } \\
\text { Interval }(\mathrm{cm})\end{array}$ & 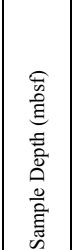 & 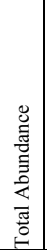 & 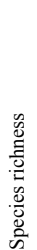 & 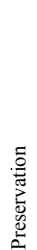 & 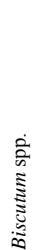 & 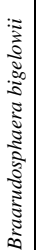 & 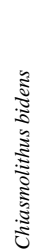 & 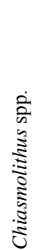 & 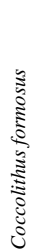 & 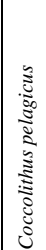 & 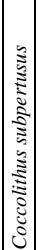 & 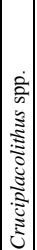 & 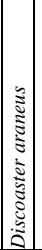 & 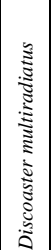 & 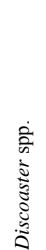 & 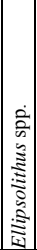 & 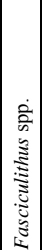 & 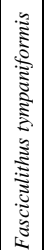 & 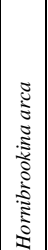 & 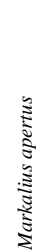 & 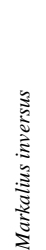 & 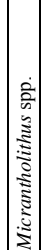 & 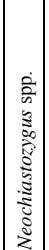 & 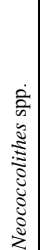 & 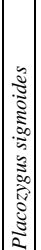 & 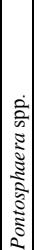 & 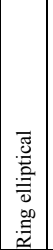 & 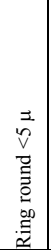 & 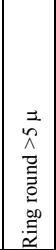 & 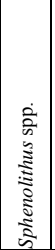 & 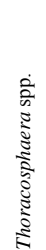 & 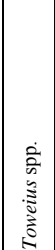 & 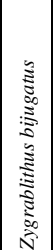 & 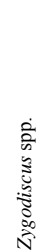 \\
\hline \begin{tabular}{|l|}
$25 \mathrm{R}-4,1-3$ \\
\end{tabular} & 233.01 & 764 & 17 & $\mathrm{P}$ & 4 & & 33 & 6 & 7 & 134 & & & & 6 & 10 & & 2 & & 2 & 8 & 41 & & \begin{tabular}{|c|}
4 \\
\end{tabular} & 2 & & & 100 & 45 & 94 & 20 & 8 & 107 & 112 & 16 \\
\hline $25 \mathrm{R}-4,5-7$ & 233.05 & 715 & 17 & $\mathrm{P}$ & 2 & & 21 & 10 & 2 & 108 & & & & 12 & 7 & & 13 & & & 9 & 43 & & 10 & 5 & 1 & & 151 & 25 & 71 & 31 & 15 & 88 & 83 & 8 \\
\hline $25 \mathrm{R}-4,7.5-9.5$ & 233.08 & \begin{tabular}{|l|}
675 \\
\end{tabular} & 17 & $P$ & 5 & & 17 & 7 & 2 & 96 & & & & 12 & 6 & & 5 & 1 & & 6 & 39 & & 11 & 9 & & & 170 & 15 & 52 & 30 & 18 & 79 & 87 & 8 \\
\hline $25 \mathrm{R}-4,10-12$ & 233.10 & 707 & 18 & $\mathrm{P}$ & 2 & & 20 & 6 & 3 & 128 & & & & 8 & 8 & & 3 & & 1 & 9 & 29 & & 2 & 9 & 3 & & 150 & 5 & 64 & 29 & 18 & 105 & 102 & 3 \\
\hline $25 \mathrm{R}-4,12-14$ & 233.12 & 711 & 16 & $P$ & 3 & & 15 & 8 & 4 & 123 & & & & 14 & 6 & & 4 & & & 7 & 32 & & 2 & 10 & & & 171 & 10 & 72 & 26 & 13 & 93 & 95 & 3 \\
\hline $25 \mathrm{R}-4,14-16$ & 233.14 & 746 & 19 & $\mathrm{P}$ & 4 & & 19 & 8 & 3 & 104 & & 2 & & 13 & 8 & 1 & 5 & & 3 & 4 & 37 & & 1 & 14 & & & 206 & 7 & 61 & 30 & 11 & 95 & 106 & 4 \\
\hline $25 \mathrm{R}-4,16-18$ & 233.16 & \begin{tabular}{|l|}
789 \\
\end{tabular} & 20 & $P$ & 3 & & 22 & 10 & 1 & 120 & & 4 & & 11 & 11 & 1 & 2 & 1 & 1 & 10 & 42 & & 2 & 10 & & & 226 & 9 & 72 & 22 & 11 & 106 & 88 & 4 \\
\hline $25 \mathrm{R}-4,18-20$ & 233.18 & 846 & 18 & $\mathrm{P}$ & 2 & & 26 & 10 & 1 & 141 & & 7 & & 8 & 6 & & 5 & & & 15 & 54 & & 10 & 10 & 7 & & 198 & 16 & 80 & 26 & 14 & 134 & 71 & 5 \\
\hline $25 \mathrm{R}-4,20-22$ & 233.20 & \begin{tabular}{|l|}
923 \\
\end{tabular} & 21 & $P$ & 2 & 1 & 21 & 8 & 5 & 146 & & 9 & & 9 & 12 & 1 & 5 & 2 & & 17 & 57 & & 5 & 11 & 6 & & 237 & 21 & 95 & 30 & 9 & 129 & 82 & 3 \\
\hline $25 \mathrm{R}-4,22-24$ & 233.22 & 672 & 17 & $P$ & & & 23 & 4 & 3 & 97 & & 2 & & 12 & 4 & & 5 & & & 10 & 40 & & 3 & \begin{tabular}{l|l}
11 \\
\end{tabular} & 2 & & 153 & 14 & 76 & 22 & 15 & 87 & 89 & \\
\hline $25 \mathrm{R}-4,24-26$ & 233.24 & \begin{tabular}{|l|}
565 \\
\end{tabular} & 18 & $P$ & 1 & & 15 & 4 & 2 & 89 & & 3 & & 7 & 3 & & 3 & 1 & & 5 & 25 & & 3 & 8 & & & 144 & 10 & 55 & 25 & 12 & 81 & 68 & 1 \\
\hline $25 \mathrm{R}-4,28-30$ & 233.28 & 613 & 19 & $\mathrm{P}$ & & & 17 & 4 & 6 & 103 & & 2 & & 10 & 5 & 1 & 8 & 1 & & 5 & 34 & & 2 & 8 & 1 & & 134 & 12 & 62 & 21 & 13 & 96 & 66 & 2 \\
\hline $25 \mathrm{R}-4,30-32$ & 233.30 & 946 & 19 & $\mathrm{P}$ & 1 & & 20 & 8 & 3 & 153 & & 2 & & 5 & 3 & 2 & 8 & & & 19 & 67 & & 5 & 11 & 8 & & 244 & 39 & 100 & 14 & 12 & 144 & 70 & 8 \\
\hline $25 \mathrm{R}-4,32-34$ & 233.32 & 737 & 20 & $P$ & 1 & & 17 & 7 & 3 & \begin{tabular}{|l|}
114 \\
\end{tabular} & & 2 & & 17 & 4 & 1 & 5 & 1 & & 14 & 41 & & 4 & 8 & 4 & & 179 & 15 & 97 & 20 & 10 & 101 & 62 & 10 \\
\hline $25 \mathrm{R}-4,34-36$ & 233.34 & 743 & 18 & $P$ & & & 13 & 8 & 4 & 102 & & 8 & & 8 & 1 & & 10 & 2 & & 16 & 53 & & 1 & 6 & 6 & & 212 & 28 & 85 & 25 & 7 & 89 & 58 & 1 \\
\hline $25 \mathrm{R}-4,36-38$ & 233.36 & \begin{tabular}{|l|}
657 \\
\end{tabular} & 18 & $P$ & 1 & & 17 & 11 & 3 & 88 & & 8 & & 9 & 2 & & 10 & 2 & & 10 & 47 & & & 9 & 1 & & 188 & 16 & 81 & 23 & 7 & 72 & 49 & 3 \\
\hline $25 \mathrm{R}-4,38-40$ & 233.38 & 886 & 20 & $P$ & 1 & & 19 & 15 & 5 & \begin{tabular}{|l|}
117 \\
\end{tabular} & & 7 & & 11 & 3 & 1 & 8 & 2 & & 18 & 74 & & 7 & \begin{tabular}{l|l}
12 \\
\end{tabular} & 2 & & 241 & 18 & 111 & 26 & 8 & 104 & 72 & 4 \\
\hline $25 \mathrm{R}-4,40-42$ & 233.40 & 775 & 20 & $\mathrm{P}$ & 2 & & 20 & 2 & 3 & 112 & & & & 10 & 6 & 1 & 9 & 3 & 1 & 13 & 47 & & 4 & \begin{tabular}{l|l}
8 \\
\end{tabular} & 4 & & 222 & 10 & 101 & 23 & 9 & 97 & 65 & 3 \\
\hline $25 \mathrm{R}-4,42-44$ & 233.42 & \begin{tabular}{|l|}
753 \\
\end{tabular} & 18 & $P$ & & & 19 & 9 & 5 & 103 & & 5 & & 7 & 2 & & 7 & 1 & & 16 & 50 & & 5 & 7 & 4 & & 225 & 14 & 94 & 24 & 8 & 87 & 59 & 2 \\
\hline $25 \mathrm{R}-4,44-46$ & 233.44 & 791 & 19 & $\mathrm{P}$ & 1 & & 27 & 9 & 3 & 112 & & 3 & & 12 & 4 & & 12 & 3 & & 11 & 51 & & 5 & 11 & 2 & & 227 & 8 & 107 & 16 & 8 & 92 & 64 & 3 \\
\hline $25 \mathrm{R}-4,46-48$ & 233.46 & 847 & 20 & $P$ & 2 & & 27 & 10 & 6 & 114 & & 7 & & 11 & & 1 & 16 & 5 & 1 & 12 & 53 & & 7 & \begin{tabular}{l|l}
10 \\
\end{tabular} & 6 & & 244 & 14 & 112 & 16 & 7 & 90 & 75 & 1 \\
\hline $25 \mathrm{R}-4,48-50$ & 233.48 & 781 & 20 & $\mathrm{P}$ & 4 & & 20 & 7 & 3 & 102 & & 6 & & 14 & 7 & 4 & 10 & 3 & & 13 & 46 & & 11 & \begin{tabular}{l|l}
6 \\
\end{tabular} & 2 & & 230 & 17 & $\begin{array}{ll}109 \\
\end{array}$ & 11 & 7 & 85 & 62 & 2 \\
\hline $25 \mathrm{R}-4,50-52$ & 233.50 & \begin{tabular}{|l|}
826 \\
\end{tabular} & 21 & $P$ & 3 & & 31 & 12 & 6 & 115 & & 3 & & 11 & 3 & 2 & 11 & 3 & 2 & 14 & 54 & & 10 & 6 & 6 & & 233 & 21 & 110 & 9 & 8 & 99 & 51 & 3 \\
\hline $25 \mathrm{R}-4,52-54$ & 233.52 & 794 & 22 & $\mathrm{P}$ & 2 & & 23 & 15 & 4 & 105 & & 2 & & 11 & 3 & 1 & 8 & 4 & 1 & 18 & 59 & 1 & 9 & 5 & 2 & & 225 & 20 & 112 & 13 & 9 & 87 & 52 & 3 \\
\hline $25 \mathrm{R}-4,54-56$ & 233.54 & 838 & 21 & $P$ & 3 & & 29 & 13 & 2 & 106 & & 3 & & 14 & 2 & 2 & 13 & 4 & 2 & 15 & 51 & & 3 & 7 & 4 & & 246 & 23 & 123 & 11 & 10 & 90 & 61 & 1 \\
\hline $25 \mathrm{R}-4,56-58$ & 233.56 & 781 & 18 & $P$ & & & 30 & 11 & 1 & \begin{tabular}{|l|}
100 \\
\end{tabular} & & 3 & & 14 & 3 & & 11 & 2 & & 13 & 46 & & 1 & 6 & 5 & & 227 & 22 & \begin{tabular}{ll|}
118 \\
\end{tabular} & 9 & 8 & 86 & 64 & 1 \\
\hline $25 \mathrm{R}-4,58-60$ & 233.58 & \begin{tabular}{|l|}
771 \\
\end{tabular} & 20 & $\mathrm{P}$ & 2 & & 31 & 12 & 6 & 92 & & 4 & & 13 & 7 & & 9 & 2 & & 16 & 52 & 1 & 6 & 5 & 5 & & 236 & 19 & 118 & 6 & 7 & 75 & 46 & 1 \\
\hline $25 \mathrm{R}-4,60-62$ & 233.60 & 661 & 19 & $\mathrm{P}$ & & & 29 & 9 & 4 & 83 & & 2 & & 14 & 7 & 1 & 9 & 3 & & 12 & 42 & & 3 & 4 & 1 & & 178 & 26 & 89 & 16 & 9 & 70 & 48 & 2 \\
\hline $25 \mathrm{R}-4,62-64$ & 233.62 & \begin{tabular}{|l|}
696 \\
\end{tabular} & 20 & $\mathrm{P}$ & 4 & 1 & 31 & 10 & 6 & 93 & & 4 & & 14 & 7 & & 11 & 3 & & 12 & 46 & & 2 & 8 & 5 & & 179 & 24 & 83 & 9 & 3 & 79 & 62 & \\
\hline $25 \mathrm{R}-4,64-66$ & 233.64 & 752 & 21 & $P$ & 2 & & 30 & 11 & 4 & 92 & & 2 & & 20 & 5 & 1 & 16 & 3 & 2 & 16 & 40 & & 1 & \begin{tabular}{l|l}
11 \\
\end{tabular} & 3 & & 196 & 29 & 95 & 11 & 11 & 82 & 68 & 1 \\
\hline $25 \mathrm{R}-4,66-68$ & 233.66 & 745 & 18 & $P$ & & & 38 & 13 & 2 & 126 & & 3 & & 10 & 2 & & 15 & 3 & & 13 & 45 & & 2 & 9 & 3 & & 178 & 14 & 78 & 13 & 10 & 105 & 62 & 1 \\
\hline $25 \mathrm{R}-4,68-70$ & 233.68 & 813 & 19 & $\mathrm{P}$ & & & 36 & 11 & 4 & 116 & & 7 & & 13 & 2 & 1 & 12 & 4 & & 11 & 51 & & 3 & 10 & 2 & & 220 & 27 & 99 & 9 & 10 & \begin{tabular}{|l|}
96 \\
\end{tabular} & 66 & 3 \\
\hline $25 \mathrm{R}-4,74-76$ & 233.74 & 707 & 21 & $\mathrm{P}$ & & & 21 & 12 & 7 & 109 & & 6 & 2 & 3 & 2 & 2 & 12 & 2 & & 17 & 39 & & 3 & 4 & 3 & 1 & 200 & 20 & 86 & 10 & 5 & 96 & 44 & 1 \\
\hline $25 \mathrm{R}-4$ & 233.76 & \begin{tabular}{|l|}
789 \\
\end{tabular} & 19 & $P$ & & & 28 & 13 & 5 & 131 & & 2 & 2 & 9 & 3 & & 18 & 3 & & 20 & 42 & & 1 & 8 & 3 & & 193 & 27 & 81 & 9 & 9 & 115 & 66 & 1 \\
\hline $25 \mathrm{R}-4,78-80$ & 233.78 & 830 & 20 & $\mathrm{P}$ & & & 24 & 14 & 8 & 135 & & 3 & 1 & 6 & 3 & & 13 & 1 & 1 & 20 & 43 & & 2 & 7 & 3 & & 233 & 18 & 102 & 5 & 6 & 121 & 59 & 2 \\
\hline $25 \mathrm{R}-4,80-82$ & 233.80 & \begin{tabular}{|l|}
722 \\
\end{tabular} & 20 & $P$ & 2 & & 23 & 16 & 3 & \begin{tabular}{|l|}
111 \\
\end{tabular} & & 2 & 1 & 4 & 2 & & 15 & 1 & & 14 & 38 & & 2 & 7 & \begin{tabular}{|l|l}
1 \\
\end{tabular} & & 212 & 16 & 90 & 3 & 5 & 107 & 45 & 2 \\
\hline $25 \mathrm{R}-4,82-84$ & 233.82 & 756 & 20 & $P$ & 1 & & 21 & 10 & 3 & 122 & & 3 & 2 & 8 & 3 & & 17 & 2 & & 7 & 27 & & 2 & 7 & 4 & & 209 & 17 & 92 & 6 & 7 & \begin{tabular}{|l|}
119 \\
\end{tabular} & 66 & 1 \\
\hline $25 \mathrm{R}-4,84-86$ & 233.84 & \begin{tabular}{|l|}
722 \\
\end{tabular} & 21 & $P$ & 1 & & 19 & 16 & 2 & 100 & & 3 & 3 & 5 & 5 & 1 & 26 & 4 & & 5 & 40 & & 1 & \begin{tabular}{l|l}
10 \\
\end{tabular} & 2 & & 203 & 13 & 90 & 7 & 3 & \begin{tabular}{|l|}
104 \\
\end{tabular} & 58 & 1 \\
\hline $5 \mathrm{R}-4,86-88$ & 233.86 & 877 & 20 & $P$ & & & 17 & 26 & 5 & \begin{tabular}{|l|}
110 \\
\end{tabular} & & 1 & 1 & 7 & 1 & 1 & 23 & 2 & & 13 & 44 & & 3 & \begin{tabular}{l|l}
8 \\
\end{tabular} & 4 & & 273 & 17 & 131 & 8 & 2 & 113 & 66 & 1 \\
\hline $25 \mathrm{R}-4,88-90$ & 233.88 & 837 & 20 & $\mathrm{P}$ & & & 16 & 23 & 3 & 94 & & 3 & 1 & 8 & 2 & 1 & 24 & 4 & & 4 & 27 & & 2 & \begin{tabular}{l|l}
10 \\
\end{tabular} & 1 & & 292 & 25 & 130 & 3 & 4 & 102 & 57 & 1 \\
\hline $25 \mathrm{R}-4,90-92$ & \begin{tabular}{|l|}
233.90 \\
\end{tabular} & \begin{tabular}{|l|}
659 \\
\end{tabular} & 20 & $P$ & 2 & & 13 & 12 & 2 & 74 & & 3 & 1 & 6 & 4 & & 25 & 3 & & 5 & 24 & & 3 & 7 & 1 & & \begin{tabular}{|l|}
217 \\
\end{tabular} & 22 & 104 & 4 & 1 & 82 & 44 & \\
\hline $25 \mathrm{R}-4,92-94$ & 233.92 & \begin{tabular}{|l|}
689 \\
\end{tabular} & 20 & $P$ & & & 9 & 25 & 3 & 78 & & 4 & 4 & 5 & 2 & 1 & 22 & 2 & & 7 & 30 & & 2 & 8 & 2 & & 222 & 24 & \begin{tabular}{|l|}
104 \\
\end{tabular} & 7 & 1 & 73 & 54 & \\
\hline $25 \mathrm{R}-4,94-96$ & 233.94 & \begin{tabular}{|l|}
719 \\
\end{tabular} & 19 & $P$ & & & 8 & 13 & 2 & 86 & & 1 & 5 & 13 & 10 & & 43 & 30 & & 5 & 22 & & 2 & 5 & 2 & & 232 & 26 & \begin{tabular}{|l|}
91 \\
\end{tabular} & 10 & 4 & 82 & 27 & \\
\hline $25 \mathrm{R}-4,96-98$ & 233.96 & 654 & 19 & $P$ & & & 10 & 11 & & 65 & 2 & & 5 & 20 & 14 & 1 & 46 & 26 & & 2 & 18 & & 3 & 1 & 3 & & 213 & 20 & 69 & 15 & 5 & 98 & 7 & \\
\hline $25 \mathrm{R}-4,98-100$ & 233.98 & \begin{tabular}{|l|}
589 \\
\end{tabular} & 21 & $P$ & 1 & & 7 & 7 & & 60 & 1 & 3 & 3 & 21 & 13 & & 45 & 34 & 1 & 1 & 11 & & 1 & 1 & 2 & & 187 & 11 & 67 & 7 & 3 & 92 & 10 & \\
\hline $25 \mathrm{R}-4,100-102$ & 234.00 & 675 & 19 & $\mathrm{P}$ & & & 18 & 6 & & 61 & & 5 & 1 & 10 & 10 & 1 & 42 & 12 & 1 & & 22 & & 3 & 1 & 2 & & 237 & 22 & 91 & 14 & 6 & 92 & 18 & \\
\hline $25 \mathrm{R}-4,102-104$ & 234.02 & \begin{tabular}{|l|}
737 \\
\end{tabular} & 20 & $\mathrm{P}$ & & & 13 & 13 & & 66 & 1 & 2 & 3 & 18 & 13 & 3 & \begin{tabular}{|l|}
45 \\
\end{tabular} & 27 & & 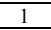 & 14 & & 2 & 1 & 1 & & 253 & 22 & 98 & 13 & 5 & 101 & 21 & 1 \\
\hline $25 \mathrm{R}-4,104-106$ & 234.04 & 639 & 19 & $\mathrm{P}$ & & & 21 & 16 & & 44 & 1 & 5 & 2 & 9 & 8 & & 33 & \begin{tabular}{|c|}
9 \\
\end{tabular} & & 2 & 15 & & 1 & 2 & 1 & & 235 & 21 & 95 & 15 & & 80 & 16 & \\
\hline $25 \mathrm{R}-4,106-108$ & 234.06 & \begin{tabular}{|l|}
760 \\
\end{tabular} & 19 & $\mathrm{P}$ & & & 31 & 15 & & 63 & 1 & 2 & 1 & 8 & 2 & & 44 & 8 & & 2 & 24 & & 2 & 3 & 1 & & 273 & 24 & 110 & 14 & 9 & 86 & 37 & \\
\hline $25 \mathrm{R}-4,108-110$ & 234.08 & 733 & 21 & $\mathrm{P}$ & & & 17 & 17 & & 60 & 1 & 1 & 2 & 8 & 6 & 2 & 30 & 15 & 1 & 1 & 20 & & 1 & 1 & 2 & & 266 & 20 & 111 & 15 & 5 & \begin{tabular}{|l|}
107 \\
\end{tabular} & 24 & \\
\hline $25 \mathrm{R}-4,110-112$ & 234.10 & \begin{tabular}{|l|}
543 \\
\end{tabular} & 19 & $P$ & & & 16 & 13 & & 47 & 2 & 1 & & 6 & 3 & 1 & 27 & $\begin{array}{ll}8 \\
\end{array}$ & 1 & 1 & 14 & & & 2 & 2 & & 197 & 15 & \begin{tabular}{|l|}
71 \\
\end{tabular} & 8 & 6 & 88 & 14 & \\
\hline $25 \mathrm{R}-4,112-114$ & 234.12 & 658 & 18 & $\mathrm{P}$ & & & 16 & 12 & & 55 & 2 & 3 & & 6 & 7 & & 26 & 12 & 1 & 2 & 23 & & & 4 & 2 & & 232 & 28 & 94 & 10 & 5 & 98 & 19 & 1 \\
\hline
\end{tabular}

already been addressed in other recent studies (see Gibbs et al. [2006] and references therein).

\section{Adaptive response of nannoplankton to the PETM}

"Excursion taxa" (ephemeral ecophenotypes restricted to the CIE; Kelly et al., 1996; Kahn and Aubry, 2004) have been interpreted as malformed nannofossils (see Jiang and Wise [2006] and references therein). Their sudden occurrence coincident with the CIE is considered to be a result of environmental-stress forcing (e.g., Jiang and Wise, 2006). Although a global distribution for these excursion taxa has been suggested (Kahn and Aubry, 2004; Raffi et al., 2005), this appears to be true only for Discoaster araneus because the other excursion taxa have not been reported in the high southern latitudes (Sites 690 and 1135). The percent abundance of $D$. araneus decreases markedly from the low to high latitudes, but could not be entirely temperaturecontrolled, taking into account rather flat equator-pole temperature gradient during the PETM and the fact that non-excursion discoasters show similar abundances at both low and high latitudes (Raffi et al., 2005; Jiang and Wise, 2006). The cause, however, still remains unknown due to limited data.

At Site 1135, nannofossil abundance patterns changed in step with changes in seawater chemistry during the development of the CIE, which further confirms that $\mathrm{K}$ - and $\mathrm{r}$-selected taxa are sensitive 
environmental indicators (e.g., Bralower, 2002). However, their extreme values preceded the peak of the CIE, and they recovered to pre-event levels ahead of the CIE (Fig. 2). This suggests that nannoplankton can adapt to changing environments and then resume normal productivity once they adjust to the environmental stresses, although the possibility of a lag in the ocean's response caused by system inertia cannot be excluded.

\section{Summary}

New and published data confirm surface-water oligotrophy in the southern high-latitude open ocean across the PETM. Depletion of nutrients culminated during the peak of the event, which may have resulted from intensified water-column stratification. Enhanced dissolution from an elevated $\mathrm{pCO}_{2}$ from the onset to the peak of the event caused a major drop in the accumulation of dissolution-susceptible holococcoliths.

Similarities and contrasts between the results of this study and previous work from open-ocean sites (ODP 690 and 1209) and shelf margins (e.g., Site 1259 and Wilson Lake, New Jersey) further demonstrate that the response to the PETM was consistent in the open oceans, but could be localized on the continental shelves where nutrient regimes depend on the local geologic setting and oceanographic conditions.

Acknowledgements. We thank James Arney for his preliminary delineation of the PETM interval we examined at Site 1135 (Arney, 2002), and Yang Wang and Yingfeng Xu for facilitating our analyses of bulk foraminiferal isotopic measurements. Co-editor Alan Cooper and referees Timothy Bralower and John Barron provided constructive and helpful comments that greatly improved this manuscript. This research used samples and data provided by ODP. Funding provided by JOI USSAC (for ODP Legs 183 and 207) and NSF-OPP grants 0126218 and 0230469 to SWW.

\section{References}

Arney, J. E. (2002), Paleocene-Eocene nannofossil biostratigraphy of ODP Leg 183, Kerguelen Plateau, Master thesis, Department of Geological Sciences, Florida State University, Tallahassee, Florida.

Aubry, M.-P., S. Lucas, and W. A. Berggren (eds.), 1998, Late Paleocene-early Eocene climatic and biotic events in the marine and terrestrial records. Columbia Univ. Press, New York, 513 pp.

Bains, S., R. M. Corfield, R. D. Norris (1999), Mechanisms of Climate Warming at the End of the Paleocene, Science, 285, 724-727.

Bains, S., R. D. Norris, R. M. Corfield, and K. L. Faul (2000), Termination of global warmth at the Palaeocene/Eocene boundary through productivity feedback, Nature, 407, 171-174.

Bown, P. R. (2005), Palaeogene calcareous nannofossils from the Kilwa and Lindi areas of coastal Tanzania (Tanzania Drilling Project sites 2003-4). J. Nannoplankton Res., 27, 21-95.

Bralower, T. J. (2002), Evidence of surface water oligotrophy during the Paleocene-Eocene Thermal Maximum: nannofossil assemblage data from Ocean Drilling Program Site 690, Maud Rise, Weddell Sea, Paleoceanogr., 17, 1023, doi: 10.1029/2001PA000662.

Chepstow-Lusty, A., N. J. Shackleton, and J. Backman (1992), Upper Pliocene Discoaster abundance variations from the Atlantic, Pacific and Indian Oceans: the significance of productivity pressure at low latitudes, Mem. Sci. Geol., 44, 357-373.

Crudeli D., J. R. Young, E. Erba, M. Geisen, P. Ziveri, G. J. de Lange, and C. P. Slomp (2006), Fossil record of holococcoliths and selected hetero-holococcolith associations from the Mediterranean (Holocene- late Pleistocene): Evaluation of carbonate diagenesis and palaeoecological-palaeocenographic implications, Palaeogeogr., Palaeoclimat., Palaeoecol., 237, 191-224.

Dickens, G. R., J. R. O'Neil, D. K. Rea, and R. M. Owen (1995), Dissociation of oceanic methane hydrate as a cause of the carbon isotope excursion at the end of the Paleocene. Paleoceanogr., 10, 965-971.

Gibbs, S., T. J. Bralower, P. R. Bown, J. C. Zachos, and L. M. Bybell (2006), Shelf and open-ocean calcareous phytoplankton assemblages across the Paleocene-Eocene Thermal Maximum: Implications for global productivity gradients, Geology, 34, 233-236.

Haq, B. U., and G. P. Lohmann (1976), Early Cenozoic calcareous nannoplankton biogeography of the Atlantic Ocean, Mar. Micropaleont., 1, 119-194.

Jiang S., and S. W. Wise (2006), Surface-water chemistry and fertility variations in the tropical Atlantic across the Paleocene/Eocene Thermal Maximum as evidenced by calcareous nannoplankton from ODP Leg 207, Hole 1259B, Rev. Micropaléont., 49(4), 227-244.

Kahn, A., and M.-P. Aubry (2004), Provincialism associated with the Paleocene/Eocene Thermal Maximum: temporal constraint, Mar. Micropaleont., 52, 117-131.

Kelly, D. C., T. J. Bralower, J. C. Zachos, I. Premoli-Silva, and E. Thomas (1996), Rapid diversification of planktonic foraminifera in the tropical Pacific (ODP Site 865) during the Late Paleocene Thermal Maximum, Geology, 24, 423-426.

Kennett, J. P., and L. D. Stott (1991), Abrupt deep-sea warming, palaeoceanographic changes and benthic extinctions at the end of the Palaeocene, Nature, 353, 225-229.

Monechi, S., E. Angori, K. von Salis (2000), Calcareous nannofossil turnover around the Paleocene/Eocene transition at Alamedilla (southern Spain), Bull. Soc. Géol. Fr., 171, 477-489.

Norris, R. D., and U. Röhl (1999), Carbon cycling and chronology of climate warming during the Palaeocene/Eocene transition, Nature 401, 775-778.

Nunes, F., and R. D. Norris (2006), Abrupt reversal in ocean overturning during the Palaeocene/Eocene warm period, Nature, 439, $60-63$.

Okada, H., 1992. Use of microbeads to estimate the absolute abundance of nannofossils. INA Newsl., 14(3), 96-97.

Raffi, I., J. Backman, and H. Pälike (2005), Changes in calcareous nannofossil assemblages across the Paleocene/Eocene transition from the paleo-equatorial Pacific Ocean, Palaeogeogr., Palaeoclimat., Palaeoecol. 226, 93-126.

Stoll, H. M., and S. Bains (2003), Coccolith $\mathrm{Sr} / \mathrm{Ca}$ records of productivity during the Paleocene-Eocene Thermal Maximum from the Weddell Sea, Paleoceanogr. 18(2), doi 10.1029/2002PA000875.

Storey, M., R. A. Duncan, and C. C. III Swisher (2007), PaleoceneEocene Thermal Maximum and the opening of the northeast Atlantic. Science, 316: 587-589.

Svensen, H., S. Planke, A. Malthe-Sorenssen, B. Jamtveit, R. Myklebust, T. R. Eidem, and S. Rey (2004), Release of methane from a volcanic basin as a mechanism for initial Eocene global warming, Nature 429: 542 -545.

Wei, W., and J. J. Pospichal (1991), Danian calcareous nannofossil succession at Site 738 in the southern Indian Ocean, in Proc. ODP, Sci. Results, J. Barron, B. Larsen, et al., 119, pp. 495-512, College Station, TX (Ocean Drilling Program).

Wei, W., and S. W. Wise (1990), Biogeographic gradients of middle Eocene-Oligocene calcareous nannoplankton in the South Atlantic Ocean, Palaeogeogr., Palaeoclimat., Palaeoecol., 79, $29-61$.

Zachos, J. C., U. Röhl, S. A. Schellenberg, A. Sluijs, D. A. Hodell, D. C. Kelly, E. Thomas, M. Nicolo, I. Raffi, L. J. Lourens, H. McCarren, and D. Kroon (2005), Rapid acidification of the ocean during the Paleocene-Eocene Thermal Maximum, Science, 308, 1611-1615.

Zachos, J. C., M. W. Wara, S. Bohaty, M. L. Delaney, M. R. Petrizzo, A. Brill,T. J. Bralower, and I. Premoli-Silva (2003), A transient rise in tropical sea surface temperature during the Paleocene-Eocene Thermal Maximum, Science, 302, 1551-1554. 